\title{
AUTODETERMINACIÓN \\ EN ADOLESCENTES Y JÓVENES \\ CON DISCAPACIDAD INTELECTUAL: \\ PERSPECTIVA DE LOS PADRES
}

\section{Self-Determination in Adolescents and Young People with Intellectual Disabilities: The Parents' Perspective}

\author{
Eliana Noemí SABEH \\ Universidad del Norte de Santo Tomás de Aquino \\ elianasabeh@gmail.com \\ Licia María Sotelo Aguilar \\ Universidad del Norte de Santo Tomás de Aquino \\ María Paula Carreras \\ Universidad Nacional de Tucumán
}

Recepción: 3 de mayo de 2020

Aceptación definitiva: 1 de julio de 2020

RESUMEN: La autodeterminación de las personas con discapacidad es esencial para el logro de su calidad de vida. El apoyo que brindan las familias a los hijos depende en buena medida de las percepciones que sostienen sobre la autodeterminación. Por eso esta investigación pretende describir la opinión de padres de adolescentes y jóvenes con discapacidad intelectual acerca de este constructo. Se conformaron 5 grupos focales, con 42 participantes. El análisis lexicométrico de las entrevistas grupales, utilizando el software Iramuteq, arrojó cuatro dimensiones: autonomía; barreras en la familia y el entorno; apoyos y oportunidades; capacidades psicológicas. Los padres desconocen el concepto de autodeterminación en toda su extensión. Lo asocian con la autonomía en el cuidado personal y en las tareas del hogar y, en menor medida, con la vida en la comunidad. Las barreras familiares son de tipo psicológico: el miedo, la sobreprotección, la subestimación y falta de confianza en las capacidades del hijo. Esto los lleva a ofrecer menos oportunidades. Consideran a la sociedad poco preparada para fomentar estos procesos. Sienten la necesidad de recibir apoyo profesional orientado a revisar sus creencias, aumentar sus conocimientos y poner en práctica estrategias que faciliten el logro de la autodeterminación en sus hijos.

Palabras clave: autodeterminación; jóvenes; discapacidad intelectual; padres; cualitativo. 
AвsTRACT: Self-determination in people with disabilities is crucial for achieving their quality of life. Support given from families to their children depends to a large extent on the reinforcement of perceptions about self-determination. In that sense, this research aims to describe the opinion of parents of adolescents and young people with intellectual disabilities about this construct. Five focus groups were formed with 42 participants. The lexicometric analysis of the group interviews using Iramuteq software yielded four dimensions: autonomy; barriers in the family and the background; support and opportunities; psychological capabilities. Parents do not acknowledge the entire concept of self-determination. They associate it with autonomy for personal care and housework and, to a lesser extent, with community life. The family barriers are psychological: fear, overprotection, underestimation and lack of trust in the child's capacity. This leads to less opportunity offer. They consider that society is poorly prepared to stimulate these processes. They feel the need to receive professional support directed to revise their beliefs, increase their knowledge and put into practice strategies to facilitate the achievement of self-determination in their children.

KEY WORDS: self-determination; young people; intellectual disability; parents; qualitative.

\section{Introducción}

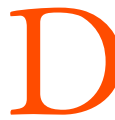

ESDE QUE SE CONSTATÓ SU VINCULACIÓN CON LA CALIDAD DE VIDA, la autodeterminación pasó a ser un concepto relevante en la investigación y en los servicios dirigidos a personas con discapacidad intelectual (Schalock, 1996; Schalock y Verdugo, 2014; Verdugo y Schalock, 2007; Verdugo, Gómez y Navas, 2013; White, Flanagan y Nadig, 2018). Quienes alcanzan mayor autodeterminación logran mejores resultados personales, en términos de inclusión laboral, relaciones sociales, participación en la comunidad, metas personales y reconocimiento de fortalezas individuales (Shogren y Ward, 2018; Shogren, Wehmeyer, Palmer, Rifenbark y Little, 2015; Wehmeyer y Bolding, 2001; Wehemeyer, Verdugo y Vicente, 2013). En definitiva, experimentan una mejor calidad de vida.

Junto con su importancia como predictora del bienestar (Wehmeyer y Field, 2007) y como dimensión en los modelos actuales de calidad de vida (Schalock y Verdugo, 2002), la autodeterminación se destacó a partir de la Convención de los Derechos de las Personas con Discapacidad, cuando se planteó el derecho a la autonomía, a la independencia y a la libertad para tomar decisiones (ONU, 2006).

Debido al auge del constructo, desde hace varias décadas se han venido desarrollando distintos modelos (Vicente, 2013; Zulueta, 2006). Dentro del campo de la discapacidad intelectual el Modelo Funcional de Autodeterminación propuesto por Wehmeyer $(1996,2001,2005,2006,2009)$ ha sido ampliamente utilizado. De acuerdo con este autor, la autodeterminación se refiere a la capacidad que desarrolla la persona para actuar como el principal agente causal de su vida, hacer elecciones y tomar decisiones. Es una característica disposicional que se refleja en las acciones cuando: 1. La persona actúa autónomamente. 2. El comportamiento es autorregulado. 3. La persona inicia y responde a los eventos con empoderamiento. 4. La persona actúa de manera autorrealizada. 
Estas cuatro características esenciales surgen del aprendizaje de un conjunto de componentes (capacidad para hacer elecciones; capacidad para tomar decisiones; habilidad de solución de problemas; habilidades para el establecimiento de metas; habilidades de autobservación, autoevaluación y autorrefuerzo; habilidad para realizar autoinstrucciones; habilidades de defensa y liderazgo) por la interacción con el entorno. Así, en el desarrollo de la autodeterminación de las personas con discapacidad entran en juego tres factores: las capacidades y habilidades de la persona, las cuales se desarrollan desde la infancia hasta la vida adulta; las oportunidades que tiene la persona para aprender esas habilidades y ponerlas en práctica día a día; y, por último, los apoyos necesarios para facilitar estos aprendizajes. Un ambiente enriquecedor que le permita poner en marcha dichas competencias y aprender otras nuevas, que le proporcione apoyos y que le dé oportunidades para practicar las habilidades adquiridas supondrá un contexto favorecedor para el desarrollo de la autodeterminación (Vicente, Guillén, Verdugo y Calvo, 2018).

Sin desconocer todo lo que se ha logrado en estas décadas de importantes avances y cambio de paradigma, aún hoy existen barreras para ejercer plenamente los derechos. Por ejemplo, estudios en los que se compara la percepción de oportunidades de autodeterminación en personas con y sin discapacidad señalan diferencias a favor de los últimos, lo que indica que la igualdad de derechos aún no se ha alcanzado (Mumbardó-Adam, Guàrdia-Olmos y Giné, 2018). Al mismo tiempo, las personas con discapacidad intelectual se perciben a sí mismas con capacidad para ejercer la autodeterminación, es decir, que se sienten capaces de elegir, tomar decisiones, resolver problemas, y encuentran las principales limitaciones en el contexto familiar, por las restricciones que les imponen para una vida independiente, junto a limitaciones en el ámbito social, como la falta de oportunidades y apoyos (Mumbardó-Adam et al., 2018; Vega, Álvarez y Jenaro Río, 2017). Esta percepción de las personas con discapacidad va en línea con los modelos actuales, cuando postulan que todas las personas tienen el potencial de ser autodeterminadas. En ese sentido, se podría argumentar que la percepción de los participantes en estos estudios alude a ese potencial.

El ámbito familiar, sin duda, juega un papel fundamental en el logro de la autodeterminación de los jóvenes con discapacidad intelectual. Los diferentes estilos parentales pueden tener influencia sobre el desarrollo de esta competencia, en función de las pautas de enseñanza que aplican con sus hijos.

Como sabemos, la familia constituye el contexto natural donde las personas se desarrollan y es en el seno de la misma donde se generan las principales estrategias para el fomento o la restricción de la autodeterminación. Si bien son aún escasos, los estudios que incluyen a la familia se han ido incrementado en los últimos años. El análisis de los mismos indica que las actitudes y creencias de los padres condicionan el desarrollo de conductas de autodeterminación en los hijos (Álvarez, Vega, Spencer, González y Arriaga, 2019; Arellano, 2015; Arellano y Peralta, 2013; Carter, Trainor, Owens, Sweden y Sun, 2009). Así, por ejemplo, cuando los progenitores creen en las posibilidades de sus hijos para la autodeterminación, la frecuencia de conductas autodeterminadas en los mismos aumenta. 
El estudio realizado por Arellano y Peralta (2013) pone en evidencia las típicas barreras familiares. Casi la mitad de los familiares encuestados suelen elegir por sus hijos; a su vez, reconocen que no fomentan el autoconocimiento de la persona con discapacidad ni la interacción con su medio, dado que no toman parte en las decisiones familiares. Tratan de presentar objetivos fáciles a sus hijos con discapacidad, evitando así la exposición al fracaso y la frustración. La sobreprotección aparece como un factor que dificulta la toma de decisiones, así como el desarrollo de competencias para la resolución de problemas. Reflejan sentir miedos diversos y la percepción de sus hijos como necesitados de protección.

Lo común a las investigaciones centradas en la familia es la necesidad de recibir orientaciones sobre estrategias que ellas puedan implementar para favorecer conductas autodeterminadas en sus hijos.

Fomentar el desarrollo de la autodeterminación, desde la infancia hasta la edad adulta, sigue siendo hoy en día un reto que implica modificar creencias familiares, actitudes sociales, ofrecer oportunidades y dar apoyos (Vicente, Mumbardó-Adam, Coma y Verdugo, 2018; Wehmeyer, Shogren, Little y López, 2017).

Es fundamental involucrar a los padres en el proceso de enseñanza/aprendizaje de la autodeterminación. Hay que indagar cómo vive la autodeterminación cada familia, desde su realidad particular, para poder luego avanzar en propuestas de utilidad encaminadas al logro de esta meta.

El objetivo de la presente investigación fue conocer la perspectiva de los padres acerca de la autodeterminación de sus hijos con discapacidad intelectual, de entre 13 y 30 años, en etapa de transición hacia la vida adulta. Se exploraron los conocimientos que poseían sobre el constructo, las barreras y oportunidades percibidas en el contexto tanto familiar como social, las necesidades de apoyo y las capacidades que ven en sus hijos para alcanzar esta meta.

\section{Método}

\subsection{Diseño}

Tomando como marco la metodología cualitativa, nos basamos en un diseño fenomenológico.

\subsection{Participantes}

Se contactó a escuelas especiales y centros de día para personas con discapacidad intelectual en el sur de la Provincia de Tucumán, Argentina. A través de estas instituciones se invitó a los padres a participar. Acudieron de forma voluntaria, por lo que se trata de una muestra de conveniencia e incidental.

Los participantes fueron 42 padres de adolescentes y jóvenes con discapacidad intelectual (la edad de los hijos osciló entre los 13 y los 30 años), que se distribuyeron en cinco grupos focales. 


\section{AUTODETERMINACIÓN EN ADOLESCENTES Y JÓVENES CON DISCAPACIDAD INTELECTUAL: PERSPECTIVA DE LOS PADRES \\ ELIANA NOEMÍ SABEH, LICIA MARÍA SOTELO AGUILAR Y MARÍA PAULA CARRERAS}

Los padres tenían entre 30 y 50 años, 4 hombres y 38 mujeres. Las familias pertenecían a un nivel socioeconómico cultural medio-bajo, provenientes de zonas rurales y urbanas, evidenciando diferentes estilos de vida y niveles educativos.

\subsection{Técnica de recolección de datos}

Se utilizó el grupo focal como técnica de recolección de datos. Consiste en una entrevista grupal, semiestructurada, que gira alrededor de una temática determinada (Escobar y Bonilla Jiménez, 2011). El objetivo de los grupos focales es que se reflejen los sentimientos, experiencias, creencias y actitudes de los participantes (Gibb, 1997). Se privilegia la profundidad de la información obtenida a la cantidad de personas que puedan participar. En este sentido, los resultados no son generalizables.

Se redactaron 28 preguntas para guiar la discusión grupal, que respondían a las siguientes dimensiones: 1) Conocimiento del concepto; 2) Capacidades del hijo; 3) Barreras en la familia y en el entorno; 4) Oportunidades de la familia y del entorno; y 5) Apoyos y necesidades de apoyos en la familia y en el entorno. Aunque en la entrevista se partió de un guion, el orden de las preguntas elaborado inicialmente fue variando para seguir el hilo de la misma, así como para matizar, profundizar o aclarar el significado de las respuestas. La duración de las entrevistas grupales fue de dos horas aproximadamente.

Las categorías iniciales que nos guiaron en las preguntas de la entrevista grupal fueron posteriormente reevaluadas y redefinidas a partir del análisis de los datos textuales realizado por el programa Iramuteq.

\subsection{Análisis de datos}

En primer lugar, se transcribieron las discusiones grupales, de audio a texto. Posteriormente, se procesó el texto en el formato requerido por el software Iramuteq. Este software realiza un análisis lexicométrico del corpus textual, es decir, un análisis cuantitativo y multidimensional de los textos. Para ello toma en cuenta la coocurrencia de varias palabras principales en un mismo enunciado, efectuando un análisis estadístico de la organización y distribución de la sucesión de palabras de un texto (Molina, 2017). Llevamos a cabo varios análisis con este programa, de los cuales presentamos uno: el análisis de clústeres o $\mathrm{CDH}$. El objetivo del $\mathrm{CDH}$ es la formación de grupos a partir del vocabulario del texto, con la característica de ser homogéneos entre los elementos que lo conforman, pero a la vez lo más diferentes a otros grupos formados. A los grupos formados (dimensiones), les dimos una denominación según las palabras que forman el contenido de los mismos y el marco teórico que guio esta investigación. El software organiza el análisis de los datos en un dendograma que ilustra las relaciones entre dimensiones. 


\section{AUTODETERMINACIÓN EN ADOLESCENTES Y JÓVENES CON DISCAPACIDAD INTELECTUAL: PERSPECTIVA DE LOS PADRES \\ ELIANA NOEMÍ SABEH, LICIA MARÍA SOTELO AGUILAR Y MARÍA PAULA CARRERAS}

\section{Resultados}

De acuerdo con el análisis de clústeres o $\mathrm{CDH}$, los datos textuales se agruparon fundamentalmente en cuatro clases, que pueden observarse en el dendograma (Figura 1).

Figura 1. Dendograma del análisis de clústeres de datos textuales de grupos focales

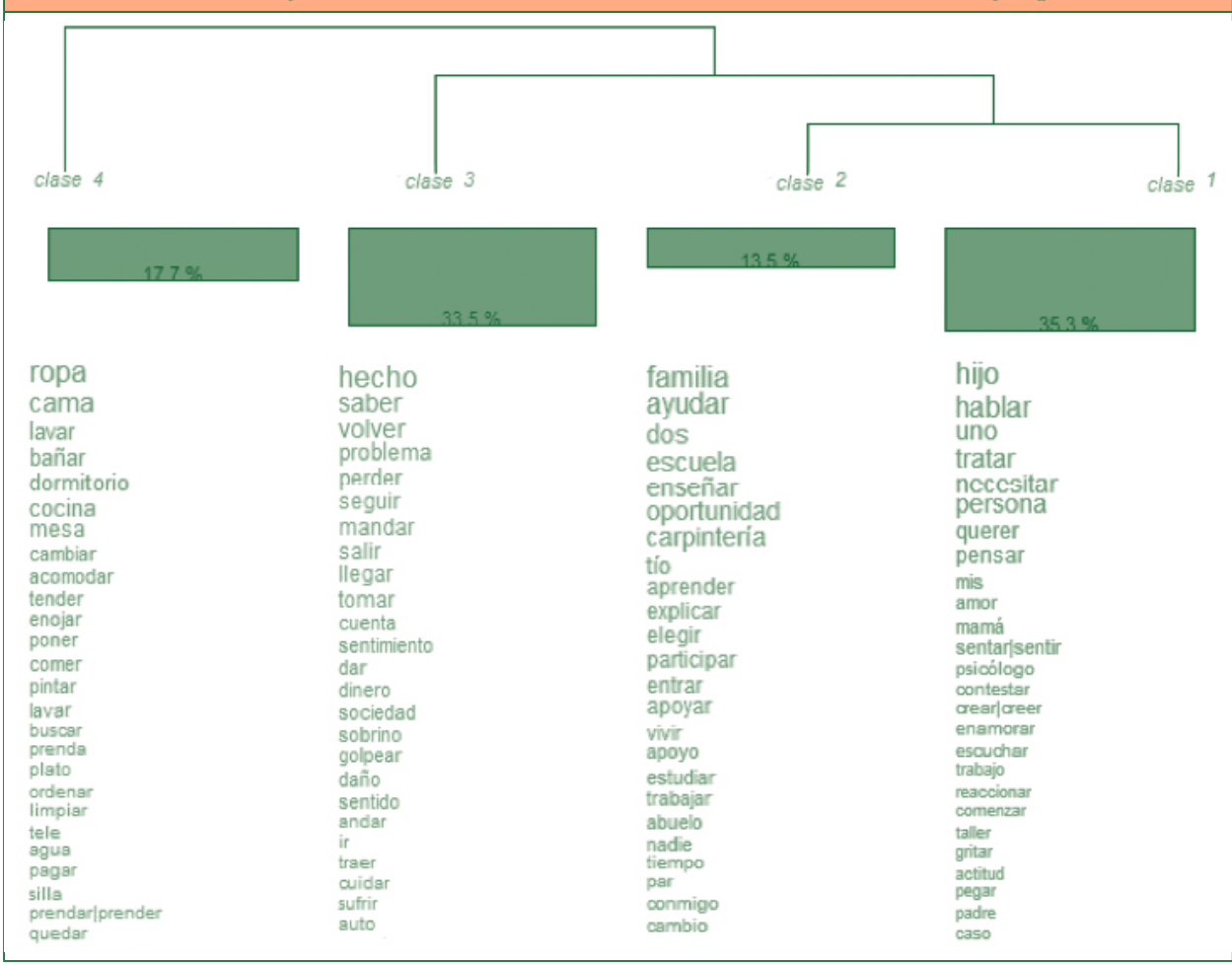

Nominamos cada clase o dimensión en función de su contenido específico: 1. Autonomía; 2. Barreras en la familia y el entorno; 3. Apoyos y oportunidades; 4. Capacidades psicológicas.

\subsection{Autonomía}

El concepto de autonomía es el más presente cuando se habla de autodeterminación de los hijos. En general, los padres de este estudio no conocen el concepto de autodeterminación. Pero están familiarizados con el de autonomía y lo relacionan con aquel: "No hemos oído hablar de autodeterminación, pero sí entendemos a lo que se refiere, más o menos lo que es, es como autonomía”. 


\section{AUTODETERMINACIÓN EN ADOLESCENTES Y JÓVENES CON DISCAPACIDAD INTELECTUAL: PERSPECTIVA DE LOS PADRES \\ ELIANA NOEMÍ SABEH, LICIA MARÍA SOTELO AGUILAR Y MARÍA PAULA CARRERAS}

Autonomía, como se observa en el dendograma (Figura 1), es una dimensión independiente de las demás, con su propio peso, que a su vez se asocia al resto de las categorías. Es decir que, desde la perspectiva de los padres, la autonomía es la idea central en relación a la autodeterminación y estaría estrechamente vinculada al resto de las categorías, esto es, barreras, apoyos, oportunidades y capacidades psicológicas.

Los vocablos que se presentaron con mayor frecuencia en esta dimensión fueron: ropa, cama, bañar, dormitorio, cocina, mesa, cambiar, acomodar, tender, poner, comer, pintar, lavar, buscar, prenda, plato, ordenar, limpiar: “A la mañana cuando se levanta me ayuda a barrer el dormitorio, me tiende las camas, me pregunta dónde va a barrer..."; "Mi hijo es independiente, él elige su ropa, se baña solo... en la casa únicamente levanta y limpia la mesa...”; "Ir al baño solos, lavarse los dientes solos, bañarse, cambiarse... algunos no lo pueden hacer solos y tienen que estar siempre acompañados por alguien"; "Es un chico que no quiere hacer nada en la casa, no se quiere bañar, no se quiere cambiar..."; "Por ejemplo, se va a la habitación, se pone a acomodar, se pone a tender la cama..."; "Ser independientes... en el caso de algunos poder ir al baño solos, lavarse los dientes solos, bañarse, cambiarse”.

Como se observa, la autonomía se vincula a realizar, con distintos grados de independencia, actividades de cuidado personal (vestirse, alimentarse, asearse) y tareas del hogar (acomodar, poner la mesa, limpiar, tender la cama, etc.). No aparecen palabras que aludan a la autonomía en el contexto social, comunitario, laboral, como podría esperarse para adolescentes y jóvenes. Es decir, sobre todo queda vinculada al hogar. "Él no se sabe manejar solo y no tiene amigos con los que comparta para salir a la calle, siempre está dentro de la casa. Sí sale con nosotros a distintos lugares, pero él todavía no. Nosotros vemos que todavía no está para largarse solo... no se maneja todavía bien con el dinero".

Por otro lado, se hace referencia a los distintos niveles de ayuda que requieren los hijos para realizar las tareas. Si bien esto es muy importante, observamos que se deja de lado un factor fundamental en la autodeterminación: si su hijo actúa de acuerdo a sus preferencias, habilidades e intereses, sin interferencias externas innecesarias, como se plantea en el modelo de Wehmeyer $(1996,2001)$. Se hace poca referencia a la idea de tomar decisiones, hacer elecciones y ser el protagonista de la propia vida. Es decir, que queda por fuera de los discursos la idea de lograr el control de su vida, tener un plan de acción, unas metas, basadas en su autoconocimiento. Podríamos concluir que, de los componentes de la autodeterminación, solo se toma en cuenta la autonomía, dejando de lado otros aspectos como autorregulación, autoconsciencia, creencia de control, planificación, acción.

\subsection{Barreras en la familia y el entorno}

Las principales palabras empleadas por los padres en esta dimensión fueron: hecho, saber, volver, problema, perder, seguir, mandar, salir, llegar, tomar, cuenta, sentimiento, dar, dinero, sociedad, sobrino, golpear, daño, sentido, andar, ir, traer, cuidar, sufrir. 
Se observa que varios términos tienen una carga negativa. Por esto los consideramos barreras para el logro de la autodeterminación. Los padres aluden a factores de la familia (incluida la familia extensa) y del entorno social más amplio: dinero, salir, andar, traer, volver, perder, seguir, mandar, sociedad, daño, traer, sufrir. "Cuando puedo hacerlo todo no la dejo que haga nada, por ejemplo, lavar no puede porque yo eso ya no la dejo, con la cocina tampoco...."; "Con mi hijo no sé qué beneficios puede tener (la autodeterminación) porque si no estamos nosotros él no avanza, por ejemplo, cuando se levanta no se cepilla los dientes si uno no lo manda, es muy dependiente"; "Por ahí capaz que sí tiene la capacidad y soy yo la que tengo miedo, entonces no sé si yo estoy muy pendiente de él o qué"; "Yo opino eso también, el problema nuestro es consentirlo o mimarlo tanto, el tema es ese, de tanto cuidarlo, quererlo, protegiéndolo, no lo dejábamos de chico que haga sus cosas, que vaya tratando de manejarse solo, porque siempre está 'No va a poder, se puede quemar, se puede caer..."; "A veces subestimamos a nuestros hijos y no les permitimos tomar decisiones en cuanto a sus acciones... hay madres que son tan sobreprotectoras que en lugar de pensar en el futuro de los chicos hacen seres inútiles, porque no tienen poder de decisión, de independencia, ni capacidades para dilucidar problemas...”.

Los padres reconocen sus propias barreras, al limitarles hacer de forma autónoma actividades para las que poseen suficiente capacidad.

Los disparadores de estas conductas, según refieren los padres, son sus propios miedos, la sobreprotección y la subestimación de las capacidades de sus hijos.

Las barreras aparecen también en relación a la familia extensa, que infantiliza al joven, siendo un obstáculo en relación a los intentos de la familia nuclear por lograr una mayor autodeterminación en los jóvenes. "Cuando vamos con mi mamá y merendamos ahí, ella sabe dónde está la taza, el azúcar, busca todo y ya sabe. Ella sola. Ahora el problema es con mi mamá que dice... 'Ay, la chiquita...' y le digo 'Ya no es chiquita', entonces me vuelve a preguntar: '¿cómo se va a poner sola?'. Y le digo: 'Déjala, sí sabe'. A veces no tengo el apoyo en general, me dicen: 'déjala a la chiquita' y ya no es chiquita"; "El apoyo de mi familia es todo al revés, porque es 'déjala es chiquita' no es el apoyo de 'déjala que haga eso y esto', es al revés, la apañan más de lo que yo hago o malcrían peor de lo que uno lo hace, pero yo sé que he tenido el apoyo de mi familia para todo...”.

Las familias reconocen la necesidad de ayuda para superar sus propias barreras: "En cuanto a la autodeterminación él está en los principios de manejarse solo, pero nosotros no tenemos todavía la capacidad de aprovechar y saber cómo ayudarlo"; "A ninguno nos han criado para que cuando tengamos nuestros hijos especiales sepamos tal o tal cosa. Nosotros lo vamos aprendiendo a medida que van creciendo, vamos aprendiendo con ellos y nos adaptamos a ellos, porque realmente no hay escuela para mamás de chicos especiales"; "Hacen mucha falta psicólogos para los padres porque hay papás que somos muy temerosos".

También se plantean la presencia de barreras en el entorno: “... la sociedad lo ha lastimado mucho a Ezequiel y me lo siguen lastimando porque no se dan cuenta de que él, por ejemplo, tiene distintos sentimientos... hay gente que no se da cuenta el 
daño que le está haciendo...”; “Lo que pasa es que la sociedad en sí no está preparada...”; “A veces los de afuera no la comprendían, ella tenía mucho conflicto en la otra escuela, le hacían burla...”.

\subsection{Apoyos y oportunidades}

Las palabras más frecuentes en esta dimensión fueron: familia, ayudar, escuela, enseñar, oportunidad, carpintería, tío, aprender, explicar, elegir, participar, entrar, apoyar, vivir, apoyo, estudiar, trabajar, abuelo, tiempo, cambio.

Se observa una polaridad positiva en la mayoría de los términos empleados. Por eso, la denominamos Apoyos y Oportunidades. Se destaca la importancia de contextos como la familia (incluida la familia extensa, ya que aparecen los términos abuelo y tío), la escuela o centro donde asisten y de estrategias de apoyo que esos contextos ofrecen para el logro de la autodeterminación, como enseñar, ayudar, explicar, apoyar, cambio. También las palabras aprender, estudiar, trabajar serían aspectos donde esos apoyos pueden resultar fundamentales. Así mismo destacamos la presencia de la palabra oportunidad, como esencial para el logro de la autodeterminación que, sumada a los apoyos, puede llevar a las personas con discapacidad a alcanzar sus metas.

Así, en relación al trabajo que realizan los centros a los que asisten, los padres manifiestan: "A ella la ayudaron mucho acá para que pueda ser más autónoma”; "Yo creo que él pudo lograr esto porque aquí lo incentivan mucho”; "La escuela está poniendo mucho, los hacen salir de viaje por dos o tres días, que también ese es un paso adelante, estar lejos de los papás”.

Con respecto al cambio de actitudes familiares que favorecen el logro de la autodeterminación, manifiestan: "Bueno, él se maneja solo y a su manera. Antes era distinto, pero ahora cambió. Si quiere comer algo abre la heladera y lo hace. Se levanta temprano, si él quiere se baña, ahora sí lo dejo que haga sus cosas solo"; "Por ejemplo, le digo que vaya a comprar el pan, y ella va y vuelve. Es lindo porque ella ya sabe que tiene que ir y volver. Ella también tiene derecho a elegir, hay que darle la prioridad de elegir y eso me gusta: que diga... me gusta esto o me gusta lo otro"; "Yo veo que cuando uno lo protege un poco menos aprende más, es como que la discapacidad no aumenta"; "Ahora ha aprendido carpintería, hizo un banco, estoy muy agradecida de la escuela"; "Mi hijo quiere ser varias cositas para futuro, quiere aprender electricidad, técnico de automotor, tiene varias cosas que me dice él que quiere aprender para el futuro. Bueno, uno lo alienta y es mejor para él, tiene toda la vida por delante”.

Por otro lado, los padres reconocen la importancia de dar apoyo psicológico a sus hijos: "Que ella sepa que cada vez puede dar unos pasitos más adelante... entonces en la medida que le das seguridad de que va a poder hacerlo bien lo va logrando, por eso es importante decirle que le va a salir bien, pasito a pasito se va logrando”; “... el amor excesivo que se confunde con compasión no lleva a nada, creo que está en exigirles y en demostrarles, porque cada cosita que ella hace y le sale bien se le refleja en la cara"; “El papá de él siempre dice: 'No, Francis no puede' y yo siempre termino peleando porque le digo: ‘¿Por qué no puede si tiene manos, ojos, como los otros también?’”. 


\subsection{Capacidades psicológicas}

En esta dimensión se agrupan palabras como hijo, hablar, uno, tratar, necesitar, persona, querer, pensar, amor, mamá, sentir, psicólogo, contestar, crear, creer, enamorar, escuchar, trabajo, reaccionar, comenzar, taller, gritar, actitud, pegar, padre, caso.

Son términos vinculados a la propia persona con discapacidad y su círculo más íntimo (hijo, persona, mamá, papá, psicólogo). En ese sentido, encontramos términos que reflejan aspectos psicológicos de la persona, implicados en el logro de la autodeterminación (pensamientos, sentimientos y conductas): pensar, sentir, crear, creer, escuchar, reaccionar, comenzar, pegar, actitud, caso, gritar, enamorar, amor: "El sueño de Johnny es casarse. El año pasado estaba muy enamorado de una chica de acá, y la escuela estaba muy revolucionada”; "Yo le digo una vez a una mamá, que tenía a la hija que lo abrazaba al chico y me decía 'A mí no me gusta eso' y le digo: 'Señora, su hija es un ser humano igual que nosotros, a Ud. le ha gustado presumir, enamorarse, tener novio, se ha casado... su hijo es igual, un ser humano'... Obvio que ella lo va a abrazar o besar y de ahí no va a pasar nada más"; "Mi niña tiene 21 años y puede caer la tormenta que caiga y no hace nada. Un día me acuerdo, yo había salido y dejé la ventana abierta en mi habitación. Se largó un viento, piedra y ella estaba a la par de la habitación y no se levantó a cerrar la ventana. Llego, y la habitación estaba inundada, pero ella no se levanta”.

La mayoría de los padres consideran que sus hijos no tienen las competencias necesarias para la autodeterminación, en especial, elegir, tomar decisiones o resolver problemas. En ocasiones matizan sus respuestas, indicando que, dependiendo del tipo de decisión o problema a resolver, posee o no la capacidad suficiente para hacerlo. Al preguntarles si sus hijos pueden elegir, tomar decisiones o resolver problemas expresan: "Yo creo que no, depende de lo que sea. Cuando él quiere hacer algo, me pregunta y según eso le digo sí o no"; "No, yo creo que no. Si se le rompe algo, por ejemplo, se asusta y tal vez puede solucionarlo después, pero problemas familiares, eso ya no”.

Los padres observan dificultades en sus hijos para expresar deseos y necesidades: "Yo decido prácticamente todo por ella, porque a mi hija le falta bastante para decir que ella decida. Recién ahora está empezando a expresarse cuando quiere algo o no, pero por el carácter de ella no es de expresarse mucho. Entonces yo le digo que me hable. Ahora empezó a llorar y a gritar y me dice la psicóloga que es la edad y empezó a rebelarse pero a mí no me gusta cómo se rebela porque ella es de poco hablar"; "Lo dejo que él se exprese porque si no le estoy cortando y nunca voy a dejar que él hable, porque siempre lo estoy defendiendo y deduzco lo que quiere decir, y mis otros hijos me dicen 'No mamá, déjalo que hable porque así no lo ayudas a él'; “Yo vengo todos los días y veo cómo los chicos se emocionan cuando algo les gusta o se ponen tristes cuando algo no les gusta. Lo expresan con su cara"; "Ella puede ser independiente en ciertas cosas, pero no para decirme qué cosas quiere para su futuro. Ahora tiene 24 años y depende mucho de nosotros".

También se identifica como limitación psicológica la inseguridad de los hijos a la hora de visualizarse a sí mismos siendo autodeterminados, con la posibilidad de una 


\section{AUTODETERMINACIÓN EN ADOLESCENTES Y JÓVENES CON DISCAPACIDAD INTELECTUAL: PERSPECTIVA DE LOS PADRES \\ ELIANA NOEMÍ SABEH, LICIA MARÍA SOTELO AGUILAR Y MARÍA PAULA CARRERAS}

vida más independiente: "Él en la casa no sabe hacer nada. Yo le digo: 'Ayúdame a hacer las camas, lavar las tazas', y me dice: 'Yo no puedo’. No es eso, no quiere. La cosa es que tenga ganas". "Yo le dije a mi hija que no voy a vivir para toda la vida. Me contesta que no le importa, porque el hermano se va a hacer cargo de ella”.

Surge también la temática de las dificultades de autodeterminación en la vida afectiva y sexual: "Los chicos con discapacidad tienen los mismos sentimientos que

\begin{tabular}{|c|c|}
\hline DOMINIO & OPINIONES DE LOS PADRES \\
\hline Autonomía & $\begin{array}{l}\text { "No hemos oído hablar de autodeterminación, pero sí entendemos a lo } \\
\text { que se refiere, más o menos lo que es, es como autonomía". } \\
\text { "Mi hijo es independiente, él elige su ropa, se baña solo... en la casa } \\
\text { únicamente levanta y limpia la mesa...”. } \\
\text { "Ir al baño solos, lavarse los dientes solos, bañarse, cambiarse... algunos } \\
\text { no lo pueden hacer solos y tienen que estar siempre acompañados por } \\
\text { alguien”. }\end{array}$ \\
\hline $\begin{array}{c}\text { Barreras } \\
\text { en la familia } \\
\text { y el entorno }\end{array}$ & $\begin{array}{l}\text { "Por ahí capaz que sí tiene la capacidad y soy yo la que tengo miedo, } \\
\text { entonces no sé si yo estoy muy pendiente de él o qué". } \\
\text { "Yo opino eso también, el problema nuestro es consentirlo o mimarlo } \\
\text { tanto, el tema es ese, de tanto cuidarlo, quererlo, protegiéndolo, no lo } \\
\text { dejábamos de chico que haga sus cosas, que vaya tratando de manejarse } \\
\text { solo, porque siempre está. 'No va a poder, se puede quemar, se puede } \\
\text { caer...”. } \\
\text { "A veces subestimamos a nuestros hijos y no les permitimos tomar } \\
\text { decisiones en cuanto a sus acciones... hay madres que son tan } \\
\text { sobreprotectoras que en lugar de pensar en el futuro de los chicos hacen } \\
\text { seres inútiles, porque no tienen poder de decisión, de independencia, ni } \\
\text { capacidades para dilucidar problemas...". }\end{array}$ \\
\hline $\begin{array}{c}\text { Apoyos } \\
\text { y oportunidades }\end{array}$ & $\begin{array}{l}\text { "Bueno, él se maneja solo y a su manera. Antes era distinto, pero ahora } \\
\text { cambió. Si quiere comer algo abre la heladera y lo hace. Se levanta } \\
\text { temprano, si él quiere se baña, ahora sílo dejo que haga sus cosas solo". } \\
\text { "Por ejemplo, le digo que vaya a comprar el pan, y ella va y vuelve. Es } \\
\text { lindo porque ella ya sabe que tiene que ir y volver. Ella también tiene } \\
\text { derecho a elegir, hay que darle la prioridad de elegir y eso me gusta: que } \\
\text { diga... me gusta esto o me gusta lo otro". }\end{array}$ \\
\hline $\begin{array}{l}\text { Capacidades } \\
\text { psicológicas }\end{array}$ & $\begin{array}{l}\text { “... Si se le rompe algo, por ejemplo, se asusta y tal vez puede solucionarlo } \\
\text { después, pero problemas familiares, eso ya no". } \\
\text { “... Yo decido prácticamente todo por ella, porque a mi hija le falta } \\
\text { bastante para decir que ella decida". } \\
\text { "Ella puede ser independiente en ciertas cosas, pero no para decirme qué } \\
\text { cosas quiere para su futuro. Ahora tiene } 24 \text { años y depende mucho de } \\
\text { nosotros”. } \\
\text { "No veo a mi hija de novia, pero los sentimientos, tienen los mismos } \\
\text { sentimientos, deseos, pero no están preparados para eso. Yo como mamá } \\
\text { veo que mi hija no está preparada para tener novio". }\end{array}$ \\
\hline
\end{tabular}


tiene cualquier otro chico de su edad, tienen los mismos sentimientos de enamorarse como cualquiera... Porque nuestro hijo es discapacitado y quizá por el solo hecho de eso no tiene derecho a enamorarse o no puede enamorarse, yo no lo veo"; "No veo a mi hija de novia, pero los sentimientos, tienen los mismos sentimientos, deseos, pero no están preparados para eso. Yo como mamá veo que mi hija no está preparada para tener novio".

Los padres refieren que sus hijos tienen metas, tienen sueños: "Ella quiere irse a vivir a Córdoba, ya ha elegido el barrio y todo, ella tiene claras las metas, sabe a dónde quiere llegar, y cuando quiere algo es más testaruda que la madre y lo hace. Cuando ella tiene ganas de hacer algo lo consigue"; "El mío también es esa persona que sabe qué es lo que quiere y hasta dónde y que puede más... Él quiere, por ejemplo, tener plata, pero únicamente para recoger todos los animales de la calle y darles de comer..."; "La mía, la Virginia, quiere ser cocinera, ella quiere llegar a ser cocinera, dice".

También identifican la falta de autorregulación como una limitación en el logro de la autodeterminación: "... yo ya le he hablado, le digo, vos no tenés que salir a pegar, te tenés que tranquilizar... ellos reaccionan como que creen que tienen superpoderes”; "Yo creo que los chicos no miden el peligro...".

\section{Discusión}

Los estudios sobre la autodeterminación de las personas con discapacidad han cobrado interés, al haberse constatado su importancia para el logro de la calidad de vida (Chao, 2018; Morán, Gómez y Alcedo, 2019; Nota, Ferrari, Soresi y Wehmeyer, 2007; Schalock, Verdugo, Gómez y Reinders, 2016; Walker y otros, 2011). Los resultados de dichos estudios indican que bajos niveles de autodeterminación impactan negativamente en la satisfacción con la vida; a su vez, un alto grado de autodeterminación se relaciona con resultados positivos en la vida adulta, tanto en lo referente a poder establecer metas personales, como en la percepción de bienestar y la inclusión social (Di Maggio, Shogren, Wehmeyer y Nota, 2020; Wehmeyer, 2020).

Se concluye, por tanto, que resulta fundamental ofrecer apoyos y oportunidades para posibilitar a las personas con discapacidad lograr el mayor desarrollo posible en autodeterminación. Es innegable que la familia constituye el entorno natural más importante para la promoción de habilidades de vida independiente.

Dado que la familia puede ser un factor facilitador o una barrera para el desarrollo de competencias de autodeterminación, hemos centrado nuestro estudio en las percepciones de padres de adolescentes y jóvenes con discapacidad intelectual. Se buscaba determinar en qué medida los padres conocían el concepto, qué barreras y oportunidades identificaban en el contexto, los apoyos que necesitaban y qué capacidades veían en sus hijos para alcanzar la autodeterminación.

Sobre el conocimiento del concepto, los datos indican en su conjunto que la mayoría de los padres no están familiarizados con él. Conocen, en cambio, el de autonomía, mencionado de manera muy frecuente en vinculación con el cuidado personal y la vida en el hogar. Se hace menos referencia a la autonomía para la vida en la comunidad 
o para el contexto social y laboral. Probablemente es en este ámbito en el que sus hijos tienen más dificultades, y también es una dificultad de los padres, por el miedo ante los riesgos que implica la salida de un ámbito protegido como la familia o los centros a los que asisten. Estos resultados son similares a los encontrados por estudios precedentes en población con distintas discapacidades (Gómez, 2010, 2014; Morán et al., 2018).

Destacamos, también, las escasas referencias a la idea de la autodeterminación como actuar de acuerdo con las preferencias, habilidades e intereses de la persona. La autorregulación, la autoconciencia, las creencias de control, el establecimiento de metas personales o la planificación están muy poco presentes en la idea de autodeterminación sostenida por los padres. El análisis lexicométrico evidencia un escaso uso de palabras como elegir, decidir. Si bien la autonomía como posibilidad de la persona de realizar el mayor número de cosas por sí sola es fundamental, que la persona tenga la opción de decidir sobre aquello que le atañe directamente es también algo esencial. Desde la perspectiva de derechos implica un empoderamiento de la persona con discapacidad. No debemos perder de vista que las limitaciones intelectuales pueden limitar la capacidad de la persona para conocer, comprender, analizar un problema y tomar una decisión. Sin embargo, es posible que esto se haga con apoyo externo. Como plantea López Mainieri (2015) “... dar una guía difiere significativamente de decidir por otro" (p. 46).

En cuanto a las barreras, los padres reconocen que están presentes en la propia familia, y las vinculan con la sobreprotección, el miedo y la subestimación de las posibilidades de los hijos. Son las barreras psicológicas, por lo tanto, las que se interponen de modo muy potente para limitar el desarrollo de la autodeterminación en los hijos (Mumbardó-Adam et al., 2018; Schorn, 2003). A conclusiones similares llegaron Mora, Córdoba y Rodríguez (2020) en un estudio cualitativo sobre las creencias familiares que excluyen a la persona con discapacidad intelectual y reducen su capacidad de autodeterminación.

Los padres tienen dudas sobre los niveles de autodeterminación que pueden alcanzar sus hijos, las estrategias que deben utilizar para promover esta competencia y los riesgos que puede implicar la autodeterminación. Todo ello limita el apoyo de las familias a la autodeterminación (Martínez-Tur et al., 2018).

Los resultados de nuestro estudio evidencian que no solo la familia nuclear, sino también la familia extensa, puede interponer barreras, al no permitir a los jóvenes desempeñarse de manera autodeterminada en los momentos compartidos con ellos.

Por último, la sociedad, según los padres, no ofrecería oportunidades a sus hijos al no estar preparada para favorecer la autodeterminación de las personas con discapacidad intelectual.

El análisis de los apoyos que identifican los padres como esenciales para sus hijos indica que estarían vinculados a la labor de los centros a los que asisten y a los profesionales que los atienden. Además, reconocen que las actitudes positivas de los padres y el apoyo psicológico por su parte son esenciales para lograr las habilidades de la autodeterminación, entendiendo que falta desarrollar este aspecto.

Se percibe, por lo tanto, que la familia y los centros no están alineados en este sentido, y que establecer estrategias comunes favorecería el uso de estrategias efectivas 
en el logro de la autodeterminación. Teniendo en consideración la importancia de los apoyos, tanto por parte de los profesionales como de la familia, Martínez-Tur et al. (2018) analizaron las actitudes de estos dos grupos en una amplia muestra de participantes, observando que los profesionales que trabajan con personas con discapacidad presentan mejores actitudes que las familias hacia la autodeterminación de las personas con discapacidad intelectual. Por otro lado, cuando existe una comunicación abierta entre estos, las actitudes tienden a converger. Las actitudes condicionan el comportamiento. En ese sentido, destacamos la importancia del diálogo entre profesionales y padres para que puedan alinearse las actitudes y ponerse en marcha planes adecuados. La cooperación entre la familia y los equipos, el diálogo abierto entre ellos resulta entonces esencial.

En cuanto a las capacidades psicológicas, consideran que los hijos poseen habilidades para desempeñarse de manera autónoma en el ámbito del hogar; sin embargo, no creen que tengan competencias para la vida en la comunidad, la toma de decisiones, la resolución de problemas o la expresión de deseos y necesidades. Aunque reconocen que sus hijos tienen deseos y metas personales. El desarrollo de estas habilidades debería promoverse desde temprana edad, a través del juego y en las actividades de la vida cotidiana, como las que señala Chu (2018) en su estudio: tener a mano sus juguetes para poder alcanzarlos, elegir con qué quiere jugar, darle en primera instancia la posibilidad de resolver por sí solo los problemas y ofrecer ayuda solo cuando sea necesario, entre otras.

Desde la perspectiva de los padres, los hijos carecen de habilidades como la seguridad en sí mismos y la autorregulación. La percepción de los padres de una baja capacidad en los hijos se encontró también en otros estudios (Mumbardó-Adam et al., 2018). Este aspecto, sin embargo, parece diferir con la visión de las propias personas con discapacidad. En el estudio citado, por ejemplo, se observa que no existen diferencias entre jóvenes con y sin discapacidad en su percepción de capacidades para las habilidades de autodeterminación, aunque sí en cuanto a oportunidades. Estas percepciones de los padres constituirían una barrera más en el camino hacia la autodeterminación de los jóvenes.

En conclusión, el estudio presentado nos permite inferir la necesidad de promover espacios de capacitación para los padres, de modo que puedan reflexionar sobre la necesidad de dar oportunidades y apoyos a sus hijos para la toma de decisiones. También resulta fundamental ayudarles a superar miedos que limitan la participación de los jóvenes en contextos menos protegidos, de orden comunitario. Por otro lado, orientarlos a reconocer las capacidades de sus hijos. En la mayoría de los estudios realizados hasta el momento, incluido el nuestro, las familias destacan la importancia de promover la autodeterminación y el deseo de que sus hijos alcancen una vida independiente (Chu, 2018), aunque reclaman orientaciones para poder llevar a cabo dicha labor.

Consideramos que un programa para formación de padres, tomando aportaciones de Field y Hoffman (2003), debería ir orientado a aumentar sus conocimientos sobre este constructo, modificar creencias que funcionan como barreras y desarrollar habilidades de apoyo a la autodeterminación de los hijos, ofreciendo oportunidades para el logro de esta meta. 


\section{AUTODETERMINACIÓN EN ADOLESCENTES Y JÓVENES CON DISCAPACIDAD INTELECTUAL: PERSPECTIVA DE LOS PADRES \\ ELIANA NOEMÍ SABEH, LICIA MARÍA SOTELO AGUILAR Y MARÍA PAULA CARRERAS}

Siendo la toma de decisiones y la posibilidad de hacer elecciones algo central en la autodeterminación, parece esencial trabajar con los padres cómo pueden ofrecer oportunidades en este sentido, cómo fortalecer psicológicamente a sus hijos para que se sientan seguros y cómo enseñarles a tomar decisiones.

El presente estudio nos permitió describir la idea de autodeterminación que poseen los padres de adolescentes y jóvenes con discapacidad intelectual y comparar dicho conocimiento con el modelo propuesto por Wehmeyer (1996, 2001, 2005). Como limitaciones de este estudio, señalamos el no haber indagado las estrategias que utilizan los padres cuyos hijos han logrado mayores niveles de autodeterminación. Tampoco se han analizado las variables que podrían estar influyendo en estas percepciones, tales como el nivel educativo, el nivel de ingresos, el grado de discapacidad de los jóvenes o los niveles de estrés familiar (Chu, 2018).

En este sentido, investigaciones futuras podrían profundizar en los factores que determinan la percepción de los padres y el efecto de la capacitación en el cambio de actitudes y en el logro de habilidades para la autodeterminación. Así mismo, sería necesario conocer qué estrategias utilizan prioritariamente los padres con una actitud positiva hacia la autodeterminación de sus hijos, desde edades tempranas y hasta la edad adulta, diferenciándolas en función del nivel evolutivo y grado de discapacidad de sus hijos. Por último, identificar qué estrategias son más eficaces desde el punto de vista profesional en la colaboración con las familias para que estas logren ofrecer apoyos sólidos para el desarrollo de la autodeterminación de los jóvenes. En definitiva, consideramos fundamental profundizar sobre las buenas prácticas de intervención familiar.

\section{Referencias bibliográficas}

Álvarez, I., Vega, V., Spencer, H., González, F. y Arriagada, R. (2019). Adultos chilenos con discapacidad intelectual: creencias, actitudes y percepciones parentales sobre su autodeterminación. Siglo Cero, 50(2), 51-72.

Arellano, A. (2015). Autodeterminación y discapacidad intelectual: un estudio piloto con padres de la asociación de Puebla (México). Revista Digital AEH, 1, 6-21.

Arellano, A. y Peralta, F. (2013). Calidad de vida y autodeterminación en personas con discapacidad. Valoraciones de los padres. Revista Iberoamericana de Educación, 63, 145-160.

Carter, E. W., Trainor, A., Owens, L., Sweden, B. y Sun, Y. (2009). Self-determination prospects of youth with high-incidence disabilities: divergent perspectives and related factors. Journal of Emotional and Behavioral Disorders, 18(2), 67-81.

Chao, P. C. (2018). Using self-determination of senior college students with disabilities to predict their quality of life one year after graduation. European Journal of Educational Research, 7(1), 1-8.

Chu, S. Y. (2018). Perspectives of Taiwanese families: a preliminary study on promoting selfdetermination skills of young children with disabilities. Early Childhood Education Journal, 46(6), 673-681.

Di Maggio, I., Shogren, K. A., Wehmeyer, M. L. y Nota, L. (2020). Self-determination and future goals in a sample of adults with intellectual disability. Journal of Intellectual Disability Research, 64(1), 27-37. https://doi-org.ezproxy.upsa.es/10.1111/jir.12696 


\section{AUTODETERMINACIÓN EN ADOLESCENTES Y JÓVENES CON DISCAPACIDAD INTELECTUAL: PERSPECTIVA DE LOS PADRES \\ ELIANA NOEMÍ SABEH, LICIA MARÍA SOTELO AGUILAR Y MARÍA PAULA CARRERAS}

Escobar, J. y Bonilla-Jiménez, F. (2011). Grupos focales: una guía conceptual y metodológica. Cuadernos Hispanoamericanos de Psicología, 9(1), 51-67. Recuperado de http://www. tutoria.unam.mx/sitetutoria/ayuda/gfocal-03122015.pdf.

Giвв, A. (1997). Focus group. Social Research Update, 5(2), 1-8.

Gómez, L. E. (2010). Evaluación de la calidad de vida en servicios sociales: validación y calibración de la Escala Gencat [Assessment of quality of life in social services: validation and calibration of the Gencat Scale] (Tesis doctoral sin publicar). Inico, Universidad de Salamanca, Salamanca, España.

Gómez, L. E. (2014). Spanish social service recipients. En A. C. Michalos (Ed.), Encyclopedia of quality of life and well-being research (pp. 6251-6258). Dordretch, Holanda: Springer.

López Mainieri, W. (2015). La autodeterminación como derecho social de las personas con discapacidad. Autonomía Personal, 17, 40-45.

Martínez-Tur, V., Estreder, Y., Moliner, C., Gracia, E., Pãtraș, L. y Zornoza, A. (2018). Dialogue between workers and family members is related to their attitudes towards self-determination of individuals with intellectual disability. Journal of Intellectual \& Developmental Disability, 43(3), 370-379. https://doi.org/10.3109/13668250.2017.1416256

Molina, J. (2017). Tutorial para el análisis de textos con el software Iramuteq. Recuperado de https://www.researchgate.net/publication/315696508_Tutorial_para_el_analisis_de_textos_con_el_software_IR AMUTEQ.

Mora, A., Córdoba, L. y Rodríguez, C. A. (2020). Resignificación de las creencias familiares para la autodeterminación del adulto con discapacidad intelectual. Revista Latinoamericana en Discapacidad, Sociedad y Derechos Humanos, 4(1), 83-104.

Morán, M. L., Gómez, L. E. y Alcedo, M. Á. (2019). Inclusión social y autodeterminación: los retos en la calidad de vida de los jóvenes con autismo y discapacidad intelectual. Siglo Cero, 50(3), 29-46. https://doi-org.ezproxy.upsa.es/10.14201/scero20195032946

Mumbardó-Adam, C., Guàrdia-Olmos, J. y Giné, C. (2018). Assessing self-determination in youth with and without disabilities: the Spanish version of the AIR self-determination scale. Psicothema, 30(2), 238-243. http://doi.org/ 0.7334/psicothema2017.349

Nota, L., Ferrari, L., Soresi. S. y Wehmeyer, M. (2007). Self-determination, social abilities and the quality of life of people with intellectual disability. Journal of Intellectual Disability Research, 51(11), 850-865.

Organización de las Naciones Unidas. (2006). Convención de los Derechos de las Personas con Discapacidad. Recuperado de https://www.un.org/disabilities/documents/convention/convoptprot-s.pdf.

Schalock, R. L. (Ed.). (1996). Quality of life, vol I. Conceptualization and measurement. Washington, DC: American Association on Mental Retardation.

Schalock, R. L. y Verdugo, M. Á. (Eds.). (2002). Handbook on quality of life for human services practitioners. Washington, DC: American Association on Mental Retardation.

Schalock, R. L. y Verdugo, M. Á. (2007). El concepto de calidad de vida en los servicios y apoyos para personas con discapacidad intelectual. Siglo Cero, 38(4), 21-36.

Schalock, R. L. y Verdugo, M. Á. (2014). Quality of life as a change agent. En R. I. Brown y R. M. Faragher (Eds.), Disability studies. Quality of life and intellectual disability: knowledge application to other social and educational challenges (pp. 19-34). New York: Nova Science Publishers.

Schalock, R. L., Verdugo, M. Á., Gómez, L. E. y Reinders, H. (2016). Moving us towards a theory of individual quality of life. American Journal of Intellectual and Developmental Disabilities, 121(1), 1-12. http://doi.org/10.1352/1944-7558-121.1.1 


\section{AUTODETERMINACIÓN EN ADOLESCENTES Y JÓVENES CON DISCAPACIDAD INTELECTUAL: PERSPECTIVA DE LOS PADRES \\ ELIANA NOEMÍ SABEH, LICIA MARÍA SOTELO AGUILAR Y MARÍA PAULA CARRERAS}

Shogren, K. A. y Ward, M. J. (2018). Promoting and enhancing self-determination to improve the post-school outcomes of people with disabilities. Journal of Vocational Rehabilitation, 48, 187-196. http://doi.org/10.3233/JVR-180935

Shogren, K. A., Wehmeyer, M. L., Palmer, S. B., Rifenbark, G. G. y Little, T. D. (2015). Relationships between self-determination and postschool outcomes for youth with disabilities. Journal of Special Education, 53, 30-41. http://doi.org/10.1177/0022466913489733

Schorn, M. (2003). La capacidad en la discapacidad. Buenos Aires: Lugar.

Vega, V., Álvarez, I. y Jenaro, C. (2017). Autodeterminación; explorando las autopercepciones de adultos con síndrome de Down chilenos. Siglo Cero, 49(2), 89-104.

Verdugo, M. Á., Gómez, L. E. y Navas, P. (2013). Discapacidad e inclusión: derechos, apoyos y calidad de vida. En M. Á. Verdugo y R. L. Schalock (Coords.), Discapacidad e inclusión. Manual para la docencia (pp. 17-41). Salamanca: Amarú.

VicENTE, E. (2013). Evaluación de la autodeterminación en adolescentes con discapacidad intelectual: análisis de factores asociados (Tesis doctoral). Instituto Universitario de Integración en la Comunidad (INICO). Universidad de Salamanca, Salamanca, España.

Vicente, E., Guillén, V. M., Verdugo, M. Á. y Calvo, M. I. (2018). El rol de los factores personales y familiares en la autodeterminación de jóvenes con discapacidad intelectual. Psicología Educativa, 24, 75-83. https://doi.org/10.5093/psed2018a13

Vicente, E., Mumbardó-Adam, C., Coma, T. y Verdugo, M. Á. (2018). Autodeterminación en personas con discapacidad intelectual y del desarrollo: revisión del concepto, su importancia y retos emergentes. Revista Española de Discapacidad, 6(2), 7-25. https://doi. org/10.5569/2340-5104.06.02.01

Walker, H. M., Calkins, C., Wehmeywe, M. L., Walker, L., Bacon, A., Palmer, S. B. y Johnson, D. R. (2011). A socio-ecological approach to promote self-determination. Exceptionality, 19(1), 6-18. http://doi.org/ 10.108/09362835.2011.537220

Wehmeyer, M. L. (1996). Self-determination as an educational outome: why is it important to children, youth and adults with disabilities? En D. J. SAnds y M. L. WehmeYer (Eds.), Selfdetermination across the life span: independence and choice for people with disabilities (pp. 17-36). Baltimore: Brooks.

Wehmeyer, M. L. (2001). Autodeterminación. Una visión de conjunto. En M. Á. Verdugo y F. B. Jordán de URríes (Coords.), Apoyos, autodeterminación y calidad de vida: Actas de las IV Jornadas Cientificas de Investigación sobre Personas con Discapacidad (pp. 113-133). Salamanca: Amarú.

Wehmeyer, M. L. (2005). Self-determination and individuals with severe disabilities: re-examining meanings and misinterpretations. Research and Practice for Persons with Severe Disabilities, 30(3), 113-120.

Wehmeyer, M. L. (2006). Factores intraindividuales y medioambientales que afectan a la autodeterminación. Siglo Cero, 37(4), 47-56.

Wehmeyer, M. L. (2009). Autodeterminación y la tercera generación de prácticas de inclusión. Revista de Educación, 349, 45-67.

Wehmeyer, M. L. (2020). Self-determination in adolescents and adults with intellectual and developmental disabilities. Current Opinion in Psychiatry, 33(2), 81-85. https://doi-org. ezproxy.upsa.es/10.1097/YCO.0000000000000576

WeHMEYER, M.L.y Bolding, N. (2001). Enhanced self-determination of adults with intellectual disability as an outcome of moving to community-based work or living environments. Journal of Intellectual Disability Research, 45(5), 371-383. http://doi.org/10.1046/j.13652788.2001.00342.x 


\section{AUTODETERMINACIÓN EN ADOLESCENTES Y JÓVENES CON DISCAPACIDAD INTELECTUAL: PERSPECTIVA DE LOS PADRES \\ ELIANA NOEMÍ SABEH, LICIA MARÍA SOTELO AGUILAR Y MARÍA PAULA CARRERAS}

Wehmeyer, M. L. y Field, S. L. (2007). Self-Determination: instructional and assessment strategies. Thousand Oaks: Corwin Press.

Wehmeyer, M. L., Verdugo, M. Á. y Vicente, E. (2013). Autodeterminación. En M. Á. Verdugo, y R. L. Schalock (Coords.), Discapacidad e inclusión. Manual para la docencia, (pp. 463-493). Salamanca: Amarú.

Wehmeyer, M. L., Shogren, K. A., Little, T. D. y López, S. J. (Eds.). (2017). Development of self-determination through the life-course. Drodrecht: Springer. https://doi. org/10.1007/978-94-024-1042-6

White, K., Flanagan, T. D. y Nadig, A. (2018). Examining the Relationship between selfdetermination and quality of life in young adults with Autism Spectrum Disorder. Journal of Developmental and Physical Disabilities, 30, 735-754. https://doi.org/10.1007/s10882018-9616-y

Zulueta, A. (2006). Modelos teóricos de autodeterminación en el ámbito de la discapacidad intelectual. En F. Peralta, M. C. González Torres e I. Torres (Coords.), Podemos hacer oír su voz: claves para promover la conducta autodeterminada (pp. 77-100). Málaga: Aljibe. 